\title{
Modulation of the Effective Viscosity of Polymer Films by Ultraviolet Ozone Treatment
}

Xuanji Yu, ${ }^{1,2}$ Anjeza Beharaj, ${ }^{3}$ Mark W. Grinstaff, ${ }^{3}$ Ophelia K. C. Tsui ${ }^{1,2,4 *}$

${ }^{1}$ Department of Physics, Boston University, Boston, MA 02215.

${ }^{2}$ Division of Materials Science and Engineering, Boston University, Boston, MA 02215.

${ }^{3}$ Department of Chemistry, Boston University, Boston, MA 02215.

${ }^{4}$ Department of Physics, Hong Kong University of Science and Technology, Clear Water Bay, Kowloon, HONG KONG.

*Correspondence to: okctsui@bu.edu.

Keywords: Polymer thin films, Effective Viscosity, Nanoconfinement, Surface modification

\begin{abstract}
Nanoconfinement of a polymer film affords a reduction of the glass transition temperature $\left(T_{\mathrm{g}}\right)$ and effective viscosity $\left(\eta_{\mathrm{eff}}\right)$. Early on, Prof. Tisato Kajiyama pioneered the idea of enhanced polymer mobility at the free surface. This concept is now well established, and accounts for the $T_{\mathrm{g}}$ and $\eta_{\mathrm{eff}}$ reductions of thin polymer films. To pay tribute to Prof. Kajiyama's seminal contribution, it is fitting to report in this special issue the use of ultraviolet ozone (UVO) to chemically modify the surface of polymer films and thereby alter their dynamical properties for the first time. Specifically, we show that with a brief exposure time of only 1.0 second under typical UVO treatment conditions, the $\eta_{\text {eff }}$ of polystyrene (PS) films supported by silica changes
\end{abstract}


from decreasing to rapidly increasing as the film thickness is decreased upon the UVO treatment. As such, the tendency of the films to spontaneously rupture on heating is significantly lessened. Considering the simplicity and low-cost of the method, our results demonstrate that UVO treatment is an attractive method to refine the dynamical properties of polymer films and improve their thermal stability. 


\section{Introduction}

Many dynamical properties of polymer films, such as the glass transition temperature $\left(T_{\mathrm{g}}\right)$ [1-9], self-diffusion coefficient $(D)$ [10-12], and effective viscosity $\left(\eta_{\mathrm{eff}}\right)$ [13-19], are different from the bulk properties as the film thickness decreases below $\sim 100 \mathrm{~nm}$. The implications of confinement-induced dynamical properties shifts as such are important from a technological standpoint [20-24] as well as from a fundamental perspective $[1,25,26]$. Though controversial at times $[10,12,19]$, the majority of results suggest that these shifts in dynamical properties originate from the polymer possessing different properties at the films' interfaces. Prof. Kajiyama was a pioneer of polymer interfaces. In the mid-1990s to early 2000s, he and coworkers published a series of inspirational papers [27-33] showing that the molecular motions near the free surface of polystyrene (PS) are significantly faster than in bulk, and are dependent upon the polymer molecular weight (MW) [28, 30, 32], MW distribution [30] and chain-end surface energy $[31,33]$. It is now widely accepted that polymer mobility near the free surface is enhanced relative to the bulk [13, 17, 34-37]. In the region near the substrate interface, however, the polymer dynamics are slower than [36] or the same as the bulk $[8,14,38,39]$, and the extent of deviation from the bulk behavior increases with the strength of the polymer-substrate interaction $[4,6,40]$.

Based on the above discussions, one envisages the possibility of controlling the properties of polymer films by locally modifying the polymer material near the interfaces. Technologically, the capability to control the properties of polymer films will enable the design of products with desirable performances. Fundamentally, by studying the correlations between the sample alterations to the property changes, one will gain insight into the mechanism underlying the confinement-induced dynamical properties shifts, which are poorly understood. 
Ultraviolet ozone treatment (UVO) [41] is a simple and cost-effective method for altering the surface chemistry of polymers. These alterations arise from chemical reactions with introduction of oxygen species to the topmost molecular layers of the specimen [41], and is described for a variety of polymers, including polydimethylsiloxane (PDMS) [42, 43], polyethylene (PE) [44], polyether ether ketone (PEEK) [44], polypropylene (PP) [45], polyethylene terephthalate (PET) [46], and polystyrene (PS) [46-48]. Upon treatment, properties of the polymer surface, such as the density, [42] $T_{\mathrm{g}},[42,47]$ adhesion, [47, 48] elastic modulus, [48] and wettability [42-48] are modulated.

Torres et al. [49] reported the first attempt to adjust the elastic modulus of polymer films by UVO in which PS with MW = 1.3 and $493 \mathrm{~kg} / \mathrm{mol}$ supported by a PDMS elastomer were studied. Before modification, the room-temperature elastic modulus (cf. the bulk $T_{\mathrm{g}}, T_{\mathrm{g}, \mathrm{bulk}} \approx 100$ ${ }^{\circ} \mathrm{C}$ ) of the films at both MWs decreased as the film thickness, $h_{0}$, was decreased below $<\sim 60 \mathrm{~nm}$. This result was attributed to enhanced surface mobility as the surface region remained rubbery even when the bulk material was in the glassy state [27-33]. Upon UVO treatment for $30 \mathrm{~s}$ or more, however, the elastic modulus of the $493 \mathrm{~kg} / \mathrm{mol}$ PS films became independent of $h_{0}$ while that of the $1.3 \mathrm{~kg} / \mathrm{mol}$ PS films continued to decrease with $h_{0}$ reduction. Torres et al. suggested that the increase in the elastic modulus of the $493 \mathrm{~kg} / \mathrm{mol}$ films upon treatment was caused by cross-linking of the surface rubbery layer, which could have much less impact on the elastic modulus when the MW was below the entanglement MW $(\approx 18 \mathrm{~kg} / \mathrm{mol}$ for PS [50]). It has also been pointed out before that PS could be cross-linked upon UVO treatment [51, 52].

In this study, we explore the use of UVO treatment to modify the $\eta_{\text {eff }}$ of PS films supported by silica. Our data show that even with a treatment time of only $\sim 1.0 \mathrm{~s}$ under UVO conditions comparable with those used by Torres et al., the thin films exhibit profound increases 
in the $\eta_{\text {eff. }}$ A layer model, consisting of a UVO modified surface layer that is more viscous than bulk and contains more mobile species in the topmost region, on top of a bulk-like layer, accurately describes the $\eta_{\text {eff }}$ data.

\section{Experimental Methods}

\subsection{Film Preparation}

Polystyrene (PS) with a weight-average molecular weight, $M_{\mathrm{w}}$, of $451 \mathrm{~kg} / \mathrm{mol}$ and a polydispersity index, PDI, of 1.06, was purchased from Scientific Polymer Products (Ontario, NY). We shall refer to this polymer as PS451K below. Single-crystal (100) silicon wafers, covered by a $100 \pm 5 \mathrm{~nm}$ thick thermal oxide layer, were purchased from Si-TECH Inc. (Topsfield, MA). To make the films, the polymer was first dissolved in toluene $(99.8+\%$, Fisher Scientific Co.) to produce solutions with various concentrations $(0.1-3.0 \mathrm{wt} \%)$, which were then filtered through a PTFE membrane filter with nominal pore size of $0.1 \mu \mathrm{m}$ (Fisher Scientific Co.). To prepare the substrates, the silicon wafers were cut into $1 \mathrm{~cm} \times 1 \mathrm{~cm}$ slides and submerged in a freshly prepared piranha solution at $130{ }^{\circ} \mathrm{C}$ for $20 \mathrm{~min}$. Afterward the slides were thoroughly rinsed in deionized water, dried by $99.99 \%$ nitrogen gas then further cleaned in oxygen plasma for $20 \mathrm{~min}$. PS451K films with various thicknesses, $h_{0}$, of 4 to $200 \mathrm{~nm}$ were deposited by spin-coating the polymer solutions onto the cleaned silica (i.e., silica-coated $\mathrm{Si}$ ) substrates at spinning speeds of $1500-4000 \mathrm{rpm}$. A single-wavelength (633 nm) Stokes ellipsometer by Gaertner Scientific Corp. (Skokie, IL) was used to determine $h_{0}$ at multiple locations on the films. The deviations in the $h_{0}$ measurements were typically less than $0.3 \mathrm{~nm}$. 
The PS films were kept under ambient conditions for more than $5 \mathrm{~h}$ to allow evaporation of the residual solvent from the films.

\subsection{Ultraviolet Ozone Treatment}

Ultraviolet ozone treatment of the polymer films took place in a commercial UVO cleaner (Procleaner ${ }^{\mathrm{TM}}$ Plus, BioForce Nanosciences Inc., Ames, IA), equipped with a mercury grid lamp with emission wavelengths between 185 and $450 \mathrm{~nm}$ and maximum intensity at 253.65 $\mathrm{nm}$. Before treatment, the UVO cleaner was preheated for $30 \mathrm{~min}$. The nominal output of the UVO lamp was $19.39 \mathrm{~mW} / \mathrm{cm}^{2}$ at a distance of $11.1 \mathrm{~mm}$. In this experiment, the distance from the grid lamp to the film surface was $7.4 \mathrm{~mm}$. The ozone was generated from atmospheric oxygen within the chamber. The UVO exposure times applied to the films for the effect of UVO treatment on $\eta_{\text {eff }}$ were 1.0 and $3.5 \mathrm{~s}$. We had also prepared PS451K films for gel permeation chromatography (GPC) studies of the MW distributions of the polymer after different UVO treatments. For these films, the UVO exposure times were 1.0, 60.0 and $120.0 \mathrm{~s}$.

\subsection{GPC Measurement}

For GPC measurements, thicker films were required to provide sufficient material to enable a GPC run. To make these films, PS451K solutions (3.0 wt\%) prepared as described above were cast onto $3 \mathrm{~cm} \times 3 \mathrm{~cm}$ pre-cleaned silica slides, and let dry under ambient conditions

for $1 \mathrm{~d}$. To determine the film thickness, the films were scratched at several locations with a razor blade, whereat the cross-sectional profile was imaged by atomic force microscopy (AFM). The AFM result gave $h_{0}=1.6 \pm 0.6 \mu \mathrm{m}$. Prior to GPC, the cast films were treated by UVO for 
times of 1.0, 60.0 and 120.0 s. An untreated film was also studied for control. To prepare the GPC samples, the films were dissolved in tetrahydrofuran (THF, HPLC grade, 99.9+\%, Fisher Scientific Co.) to make a solution of $4 \mathrm{mg} / \mathrm{mL}$ concentration. The solutions were then filtered through a PTFE membrane filter with nominal pore size of $0.2 \mu \mathrm{m}$ (Millex ${ }^{\circledR}$, EMD Millipore). GPC measurements were performed using a Rainin Instrument Co. Inc. pump equipped with an OPTILAB DSP refractive index (RI) detector. Two series-connected Jordi Gel DVB columns were employed with THF as the eluent at a flow rate of $1 \mathrm{~mL} / \mathrm{min}$ at $25^{\circ} \mathrm{C}$. The $\mathrm{MW}$ calibration curve was obtained with Agilent technologies PS standards. The $M_{\mathrm{w}}$ and PDI values of the specimen were calculated from the GPC data by using the built-in software of the instrument.

\subsection{Effective Viscosity Measurement}

The effective viscosity, $\eta_{\text {eff }}$, was measured by monitoring the temporal evolution of the surface structure of the specimen film and modeling the evolution by assuming it to originate from the dynamics of the surface capillary waves on the film $[13,15,53,54]$. More specifically, the topographical image of the film was captured at different annealing times, $t$, at a fixed temperature, $T$, by using tapping-mode AFM. Thermal annealing was conducted under a nitrogen environment to protect the film against thermal degradation. The measurements were ex situ, meaning that the films were rapidly quenched from the annealing temperature to room temperature before individual AFM imaging. The topographic data acquired were converted to its power spectral density (PSD), $A_{\mathrm{q}}{ }^{2}(t)$, as described before [13]. Because the $M_{w}(=451 \mathrm{~kg} / \mathrm{mol})$ of our films was much bigger than the characteristic MW for entanglement to be significant in the transport dynamics of our polymer (which is $M_{c} \sim 31 \mathrm{~kg} / \mathrm{mol}$ [50]), the films were 
viscoelastic. Following a linear stability calculation that assumes the lubrication approximation, one may write [55]:

$$
\begin{aligned}
& A_{q}^{2}(t)=A_{q}^{2}(0) \exp \left(2 \Gamma_{q}^{\prime} t\right)+\left(\frac{k_{B} T}{\gamma_{s} q^{2}+G^{\prime \prime}\left(h_{0}\right)}\right)\left(1-\exp \left(2 \Gamma_{q}^{\prime} t\right)\right), \\
& \text { where } \quad \Gamma_{q}^{\prime}=-M_{\mathrm{tot}} q^{2}\left[\left(\gamma_{s} q^{2}+G^{\prime \prime}\left(h_{0}\right)\right)^{-1}+\left(\frac{3 \mu_{0}}{h_{0}^{3} q^{2}}\right)^{-1}\right]^{-1} .
\end{aligned}
$$

Here, $q$ is the wave vector of the surface capillary waves, $k_{\mathrm{B}}$ is the Boltzmann constant, $T$ is the measurement temperature in degrees Kelvin, $\gamma_{\mathrm{s}}$ is the sample surface tension, and $G\left(h_{0}\right)=$ $-A /\left(12 \pi h_{0}{ }^{2}\right)$ is the interfacial potential of the film, with $A$ being the Hamaker constant published before [56]. We had also let $A$ to be a fit parameter and found that it has little impact on the fit result (data not shown). $\Gamma_{q}^{\prime}$ denotes the relaxation rate of the surface capillary wave with wavevector $q$ on a viscoelastic film having a short-time rubbery shear modulus of $\mu_{0}$ and longtime terminal-flow mobility of $M_{\text {tot }}$ [55]. The effective viscosity $\eta_{\text {eff }}$ is defined by the relation $[13,55]$

$$
\eta_{\text {eff }} \equiv h_{0}^{3} /\left(3 M_{\text {tot }}\right)
$$

Physically, $M_{\text {tot }}$ is the steady-state in-plane current density produced in a film by applying a unit pressure gradient, $\nabla P$, across it [55]. This can be expressed mathematically by $M_{t o t} \equiv\left[-\int_{0}^{h_{0}} v(z) d z / h_{0}\right] /|\nabla P|$, where $v(z)$ is the out-of-plane $(z)$ profile of the in-plane velocity of the film fluid and determined by solving the Navier-Stokes equation for steady flow in the film produced by the pressure gradient [55]. When the films are homogeneous and there is no slippage on the substrate surface, $\eta_{\mathrm{eff}}\left(h_{0}\right)$ is a constant; in all other cases, $\eta_{\mathrm{eff}}\left(h_{0}\right)$ varies with $h_{0}$ in 
general. By assuming a specific variation of the local viscosity with the film depth, $z[13-15,19]$, and/or the presence of slippage on the substrate surface [15] as such to make the resulting $\eta_{\text {eff }}\left(h_{0}\right)$ dependence matches that obtained in experiment, microscopic details about the flow dynamics in the films can be deduced.

Shown in Fig. 1(a) to 1(c) (open circles) are the time-sequenced PSDs obtained at $T=$ $172{ }^{\circ} \mathrm{C}\left(\approx T_{\mathrm{g}, \text { bulk }}+72{ }^{\circ} \mathrm{C}\right)$ from a pristine PS451K film, a PS451K film after an UVO exposure of $1.0 \mathrm{~s}$, and another film after an exposure of $3.5 \mathrm{~s}$, respectively. On comparing the data of the pristine film and the $1.0 \mathrm{~s}$ UVO exposed film, one may readily observe the $\sim 1$ order of magnitude longer evolution times exhibited by the latter, showing that the $\eta_{\text {eff }}$ of film became 10 times higher upon the $1.0 \mathrm{~s}$ UVO exposure. (It should be mentioned that films with different $h_{0}$ may also display different evolution time scales, but the two films considered have similar $h_{0}$ 's, name 20 and $23 \mathrm{~nm}$, respectively, and so the different evolution times can only be caused by the film materials possessing different average mobilities.) Next, we note that the data of all three panels (i.e., Fig.1(a) to (c)) are enveloped by the theoretical PSD of liquid films (denoted by the dashed line in individual panels) and given by $A(q)^{2}=k_{\mathrm{B}} T /\left(\gamma_{\mathrm{s}} q^{2}\right)$. This shows that the films show no sign of elastic behaviors at long times. Moreover, because the values of $\gamma_{\mathrm{s}}$ corresponding to the "liquid envelopes" found of the UVO films are the same as that of the pristine film within uncertainty [57], the surface tension of any elastic skin that might be present in the UVO treated films due to crosslinking, if present, must be small compare to the innate surface tension of the pristine polymer. From the figures, one may also observe that the solid lines describe the data well. These lines are the best fit of the data to Eq. (1). Considering that the fitted lines in all three panels were based on the same model (Eq. (1)) [55], our result thus shows 

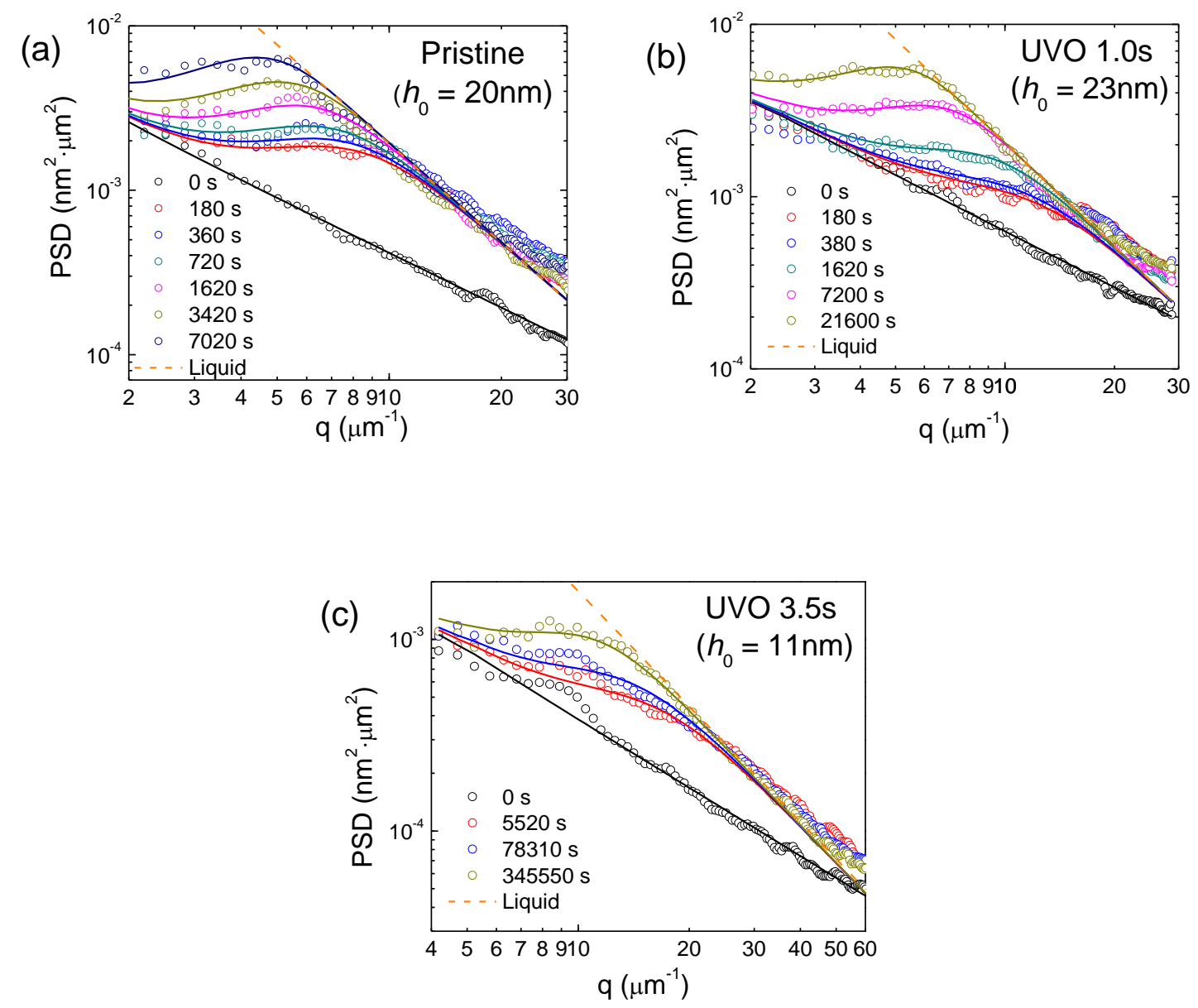

Fig. 1. Time-resolved AFM surface spectra and model fits. The open circles represent power spectral density, PSD, obtained from (a) a pristine PS451K film with $h_{0}=20 \mathrm{~nm}$ and (b) a PS451K film with $h_{0}=$ $23 \mathrm{~nm}$ after $1.0 \mathrm{~s}$ UVO exposure and (c) another PS451K film with $h_{0}=11 \mathrm{~nm}$ after $3.5 \mathrm{~s}$ UVO exposure. The measurement temperature was $T=172{ }^{\circ} \mathrm{C}$. The figure legend gives the annealing times $t$. The solid lines are model lines obtained by using Eq. (1) and assuming that (a) $\eta_{\text {eff }}=5.0 \times 10^{5}$, (b) $3.8 \times 10^{6}$, and (c) $1.8 \times 10^{8} \mathrm{~Pa} \cdot \mathrm{s}$. The dashed lines are the theoretical PSD of bulk PS liquid with surface tension assuming $\gamma_{\mathrm{s}}=0.032,0.030$ and $0.034 \mathrm{~N} / \mathrm{m}$ for panel (a), (b) and (c), respectively.

that the UVO-treated films possess the same viscoelasticity as the pristine films, namely they behave as an elastic rubber at short times but a viscous liquid at long times. 


\subsection{Complex Moduli Measurements}

The samples for complex moduli measurements were prepared by first casting PS451K solutions with a concentration of $3.0 \mathrm{wt} \%$ onto a Petri dish and allowing the cast solution to dry

for $1 \mathrm{~d}$ in a fume hood under ambient conditions. The residue was then annealed at $150{ }^{\circ} \mathrm{C}$ for 3 $\mathrm{d}$ in a vacuum oven under a pressure of 0.05 torr to be rid of the solvent. Afterward, the material was molded into disks of diameter $8 \mathrm{~mm}$ and thickness $\sim 1 \mathrm{~mm}$ in a vacuum press. If the effect of UVO was to be examined, the molded samples would be subject to UVO exposure for the desired duration. Complex moduli measurements were performed at $200{ }^{\circ} \mathrm{C}$ on a AR2000 rheometer (TA Instruments, New Castle, DE) equipped with a parallel upper plate of diameter 8 $\mathrm{mm}$ and operated in the oscillatory test mode (with $0.1 \leq \omega \leq 500 \mathrm{rad} / \mathrm{s}$ ). During measurement, the strain was fixed at $(2 \pm 0.15) \%$ and the gap between the upper plate and the lower base was maintained at $1.00 \pm 0.01 \mathrm{~mm}$. In addition, steps were taken to ensure that the sample was in the linear viscoelastic response regime when the data were taken. The Rheology Advantage Data Analysis software was used to analyze the data.

\section{Results and Discussions}

The data of $\eta_{\text {eff }}$ measured at $T=172{ }^{\circ} \mathrm{C}$ for pristine PS451K films (circles), PS451K films UVO-treated for $1.0 \mathrm{~s}$ (squares) and PS451K films treated for $3.5 \mathrm{~s}$ (triangle) are plotted against $h_{0}(\sim 5$ to $200 \mathrm{~nm})$, as shown in Fig. 2. (We shall refer to the treated films as UVO $1 \mathrm{~s}$ and UVO 3.5s, respectively, below.) As $h_{0}$ decreases below $\sim 100 \mathrm{~nm}$, the $\eta_{\mathrm{eff}}$ of the pristine films begins to decrease with decreasing $h_{0}$. But as $h_{0}$ decreases further below the radius of gyration of 
the polymer, $R_{\mathrm{g}}$, (estimated to be $\sim 18 \mathrm{~nm}$ [58]), $\eta_{\text {eff }}$ decreases much more rapidly. As we previously showed [15], this originates from enhanced mobility at the free surface that tends to

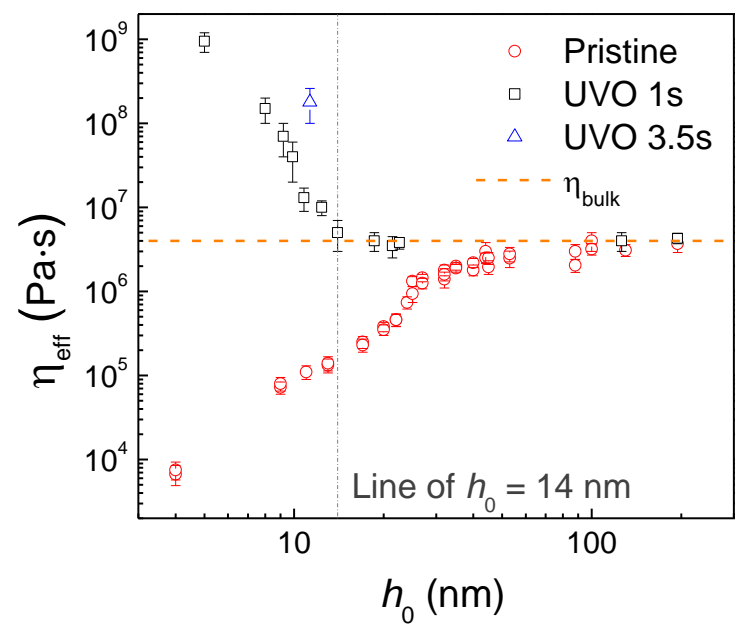

Fig. 2. Effective viscosity versus film thickness of pristine PS451K films and the PS451K films that had been exposed to UVO for $1.0 \mathrm{~s}$ and $3.5 \mathrm{~s}$. The measurement temperature was $172{ }^{\circ} \mathrm{C}$. The dashed line denotes the bulk viscosity, which equals $4.0 \times 10^{6} \mathrm{~Pa} \cdot \mathrm{s}$. The dot-dashed line indicates where $h_{0}=14 \mathrm{~nm}$ is.

cause plug flow in the films if the MW of the polymer is greater than $\sim 100 \mathrm{~kg} / \mathrm{mol}$ (which applies here) and the film thickness is less than $R_{\mathrm{g}}$. For the UVO $1 \mathrm{~s}$ films, however, the $\eta_{\mathrm{eff}}$ increases rapidly with decreasing $h_{0}$ as $h_{0}$ decreases below $\sim 14 \mathrm{~nm}$. The opposite $\eta_{\text {eff }}\left(h_{0}\right)$ dependence of the UVO 1s films relative to that of the pristine films demonstrates that UVO treatment is capable of significantly altering the flow dynamics of the films. The fact that even a mild increase of the exposure time from 1.0 to $3.5 \mathrm{~s}$ (open triangle, Fig. 2) causes the $\eta_{\text {eff }}$ to increase by more than 10 times at $h_{0} \approx 10 \mathrm{~nm}$ further documents the effectiveness of the UVO approach. 
The phenomenon of $\eta_{\text {eff }}$ increasing upon nano-confinement has been previously observed by us with unentangled poly(methyl methacrylate) (PMMA) films deposited on silica [14]. In that work, we described the $\eta_{\text {eff }}\left(h_{0}\right)$ relationship over a wide range of temperatures by using a three-layer model consisting of a bulk-like layer (where the viscosity equaled the bulk viscosity, $\eta_{\text {bulk }}$ ) sandwiched between a surface mobile layer (where the thickness was $h_{\mathrm{t}}$ and mobility was $M_{\mathrm{t}}$ ) and an immobile bottom layer (where the thickness was $h_{\mathrm{b}}$ and mobility was zero). By further assuming the no-slip boundary condition on the substrate surface, the mobility of the film was derived to be [14]:

$$
M_{\text {tot }}=\frac{\left(h_{0}-h_{b}\right)^{3}}{3 \eta_{b u l k}}+M_{t}-\frac{h_{t}^{3}}{3 \eta_{b u l k}} .
$$

In Eq. (3), $M_{\mathrm{t}} \equiv h_{\mathrm{t}}{ }^{3} /\left(3 \eta_{\mathrm{t}}\right)$, where $\eta_{\mathrm{t}}$ denotes the viscosity of the surface layer. To perceive the physical meaning of this equation, we consider the simplified case where the last term is negligible, which prevails when either $\eta_{t} \ll \eta_{\text {bulk }}$ (i.e., the surface mobility is significantly enhanced) or $h_{0}-h_{\mathrm{b}}>h_{\mathrm{t}}$ (i.e., the film thickness is large). (It should be clarified that this simplification is not applied to the measurements obtained here.) For a simplified case as such, this model (Eq. (3)) differs from the model we had used in the past to fit the data of unentangled pristine PS films on silica [13] in two respects. Firstly, the model used for unentangled PS films is a two-layer one devoid of the immobile bottom layer (and so $h_{\mathrm{b}}=0$ ). Secondly, the mobility of the surface layer, $M_{\mathrm{t}}$, in the PS film model was a constant whereas that in the PMMA film model was a function of $h_{0}$ given by [14]:

$$
M_{t}\left(h_{0}\right)=\frac{M_{t}(\infty)}{1+\exp \left[-\left(h_{0}-l_{t}\right) / \Delta l_{t}\right]},
$$


where $l_{\mathrm{t}}$ and $\Delta l_{\mathrm{t}}$ are constants. According to this equation, $M_{\mathrm{t}}$ decreases to a half of its maximum value, $M_{\mathrm{t}}(\infty)$, when $h_{0}$ decreases to $l_{\mathrm{t}}$. The sharpness of the decrease is described by $\Delta l_{\mathrm{t}}$. Thus, $l_{\mathrm{t}}$ (or more precisely, $l_{\mathrm{t}}+\Delta l_{\mathrm{t}}$ ) characterizes the film thickness whereat $M_{\mathrm{t}}\left(h_{0}\right)$ starts to decrease from $M_{\mathrm{t}}(\infty)$. Physically, introduction of the immobile bottom layer accounts for the formation of strong hydrogen bonds between the carboxylate groups present in PMMA and the silanol groups on the silica surface, which are absent in the PS films. As for the $h_{0}$ dependence of $M_{\mathrm{t}}$, it manifests due to the influence of the substrate surface on $M_{\mathrm{t}}$ when the surface layer is near the substrate surface.

We had attempted to fit the $\eta_{\text {eff }}$ data of the UVO 1s PS films to the PMMA three-layer model, but to no avail. As the dashed and dotted lines of

Fig. 3(a) show, the model produces a
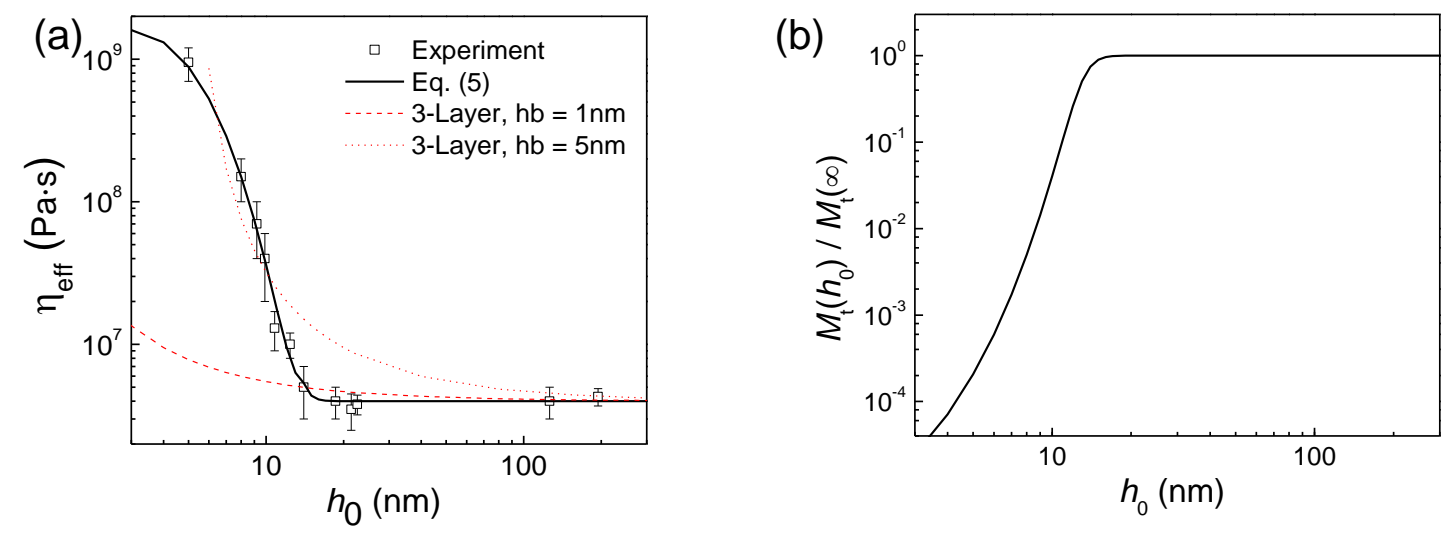
Fig. 3. (a) Effective viscosity versus film thickness of the PS451K films after $1.0 \mathrm{~s}$ UVO exposure. The dashed and dotted lines are three-layer model lines (Eq. (3)) with $M_{\mathrm{t}}$ set equal to 0 and $h_{\mathrm{b}}$, set equal to 1 and $5 \mathrm{~nm}$, respectively. The measurement temperature was $172{ }^{\circ} \mathrm{C}$. The solid line is the best fit to the UVO-layer model (Eq. (5)). In all cases, $\eta_{\text {bulk }}$ is the asymptotic value of $\eta_{\text {eff }}$ in the $h_{0}$ regime above 100 nm. (b) The functional form of the normalized surface mobility, $M_{\mathrm{t}}\left(h_{0}\right) / M_{\mathrm{t}}(\infty)$, used to calculate the solid fit line shown in Panel (a).

noticeably slower rise of $\eta_{\text {eff }}$ in the thin film region than observed in experiment. This shows that imposition of an immobile bottom layer is not sufficient to explain the abrupt rise in $\eta_{\text {eff }}$ found in the UVO 1s films upon thickness reduction to below $\sim 14 \mathrm{~nm}$. In obtaining the dashed and dotted model lines in Fig. 3(a), we assumed the thickness of the immobile bottom layer, $h_{\mathrm{b}}$, to be 1 and $5 \mathrm{~nm}$, respectively, and treated the surface mobile layer to be absent and so $M_{\mathrm{t}}=0$. Had the value of $M_{\mathrm{t}}$ been given a nonzero value, $\eta_{\mathrm{eff}}$ would become smaller and deviate further from the experimental data. We thus conclude that the PMMA three-layer model is unsuitable for describing the $\eta_{\text {eff }}$ data of our UVO treated films.

To formulate a suitable model, we reviewed the effects of UVO on PS. X-ray Photoelectron Spectroscopy (XPS) revealed that the atomic concentration of oxygen increased linearly with exposure time initially then saturated [59]. The oxygen concentration at saturation in that study $(\sim 35 \%)$ coincided with that reported in another study [47]. In the present work, the same UVO cleaner and similar UV-sample distance was employed as in the latter study. These observations suggest that the UVO conditions we applied are typical, and the UVO modifications discussed in the literature (notably Refs. [47] and [59]) should apply to our films. In Ref. [59], high-resolution analyses of the C-1s XPS peak of 180 s exposed UVO PS showed emergence of 
new peaks corresponding to $\mathrm{O}-\mathrm{C}=\mathrm{O}, \mathrm{C}=\mathrm{O}, \mathrm{C}-\mathrm{OR}$ and $\beta$-shifted $\mathrm{C}-\mathrm{C} / \mathrm{C}-\mathrm{H}$ bonds (due to juxtaposition of carbons bonded to oxygen.) At the same time, the area of the $\pi$ - $\pi^{*}$ shake-up peak, corresponding to the $\mathrm{C}-\mathrm{C}$ bonds inside a phenyl ring decreased. These data suggest breakage of some phenyl rings. However, for the exposure times used in the current experiment, i.e., 1.0 and $3.5 \mathrm{~s}$, the oxygen concentrations, as inferred from the data of Ref. [59], were quite small, $<\sim 1$ and $<\sim 3.5 \%$, respectively. In addition, the $\pi-\pi^{*}$ peak areas were reduced by only $\sim 6$ and $\sim 20 \%$, respectively, suggesting that the majority of the phenyl rings were not broken.

Additional information about UVO PS was revealed through studying the surface morphology change $[47,52]$ after samples were washed by water $[59,60]$ or ethanol $(70 \%$ in aq.) [60]. It was found that the surface of as-treated UVO PS developed sub-micron sized grains. Washing by water removed some of the grains and rendered the surface smoother [52]. It was thus proposed that the topmost portion of UVO PS contains partially fragmented oxidized chains $[52,60]$. That the top surface of UVO treated PS is populated by lower MW oxidized species also corroborates with surface mechanical measurements by atomic force microscopy (AFM) where reduced elastic modulus was found in the near surface region (several nanometers) of UVO treated PS films $[47,48]$. It also corroborates with lowering of the surface $T_{\mathrm{g}}$ as inferred from the improved ease of thermal bonding of PS microchannels upon UVO treatment [47, 48, $52]$.

Based on the above discussions about how UVO modifies PS and our results, we propose a model to explain our $\eta_{\text {eff }}$ data of the UVO 1s treated films. The observation that the $\eta_{\text {eff }}$ data overlaps with the bulk value for $h_{0}>\sim 14 \mathrm{~nm}$ indicates that the effect of UVO modifications on the rheological property of the polymer is present within $\sim 14 \mathrm{~nm}$ of the free surface (Fig. 2). This finding is consistent with the storage and loss moduli of a $\sim 1 \mathrm{~mm}$ thick PS451K specimen being 
essentially unaltered by $1.0 \mathrm{~s}$ of UVO exposure (Fig. 4), and thus the modified region should be much thinner than $1 \mathrm{~mm}$.

The model we propose to describe our $\eta_{\text {eff }}$ data is illustrated below in Fig. 5. In this model, a characteristic thickness, $h_{\mathrm{UVO}}$, is introduced to denote the spatial extent by which the UVO treatment modifies the rheological property of the polymer. Correspondingly, the films with $h_{0} \leq h_{\mathrm{UvO}}$ are fully modified. In line with the above discussions [47, 52, 59, 60], the films with $h_{0} \leq h_{\mathrm{UVO}}$ are heterogeneous, composed of low-MW species at the top and possibly mildly

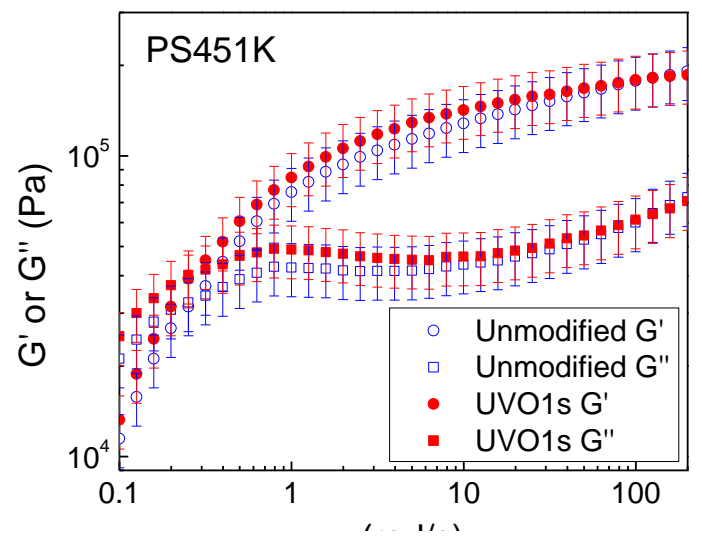

Fig. 4. Storage and loss modulus $G^{\prime}$ and $G^{\prime}$, respective, versus oscillatory frequency at $200{ }^{\circ} \mathrm{C}$ of UVO $1.0 \mathrm{~s}$ and unmodified PS451K with $h_{0} \sim 1000 \mu \mathrm{m}$.

(a) $h_{0} \leq h_{U v o}: \quad$ Low-MW surface species with total mobility $M_{\text {, }}$ OSP
Substrate

(b) $h_{0}>h_{\text {uvo: }}$

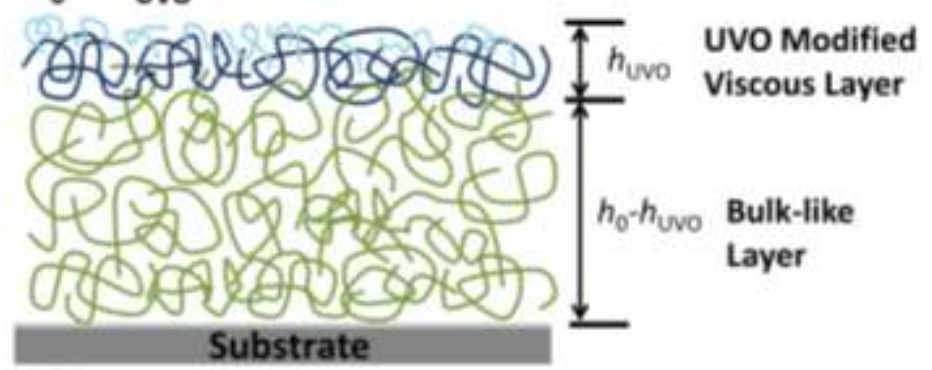


Fig. 5. Schematic representation of the layer model used to describe the UVO-modified films. (a) For the films with $h_{0} \leq h_{\mathrm{UvO}}$, the rheological property of the whole film is modified with the uppermost region made up of low-MW species that dominates the mobility of the whole film. We surmise that the modified material is more viscosity than the unmodified one which accounts for the rise in $\eta_{\text {eff }}$ found in the thin film regime. (b) For the thicker films with $h_{0}>h_{\mathrm{UVO}}$, the region within a thickness of $h_{\mathrm{UVO}}$ of the free surface is modified by UVO. It has the same structure as portrayed in (a). Beneath this layer is a bulk-like layer.

crosslinked polymer beneath (Fig. 5(a)). Given that the $\eta_{\text {eff }}$ of the UVO modified thin films are larger, the modified polymer is more viscous than the unmodified one. For $h_{0}>h_{\mathrm{UVO}}$, the film possesses an UVO-modified upper layer similar to that in Fig. 5(a) with a thickness of $h_{\text {Uvo. }}$ Below this layer is a bulk-like layer where the polymer property is similar to that of the unmodified polymer (Fig. 5(b)). Based on these descriptions, we expressed $M_{\text {tot }}$ by the following equations for different $h_{0}$ regimes:

$$
M_{\text {tot }}= \begin{cases}M_{t} & \left(h_{0} \leq h_{U V O}\right) \\ M_{t}-\frac{h_{U V O}^{3}}{3 \eta_{\text {bulk }}}+\frac{h_{0}^{3}}{3 \eta_{\text {bulk }}} & \left(h_{0}>h_{U V O}\right)\end{cases}
$$

where $\eta_{\text {bulk }}=4.0 \times 10^{6} \mathrm{~Pa} \cdot \mathrm{s}$ is the $\eta_{\mathrm{eff}}$ of pristine PS films with $h_{0}>100 \mathrm{~nm}$ and $M_{\mathrm{t}}$ is $h_{0}$ dependent given by Eq. (4). These equations describe our data well (solid line of Fig. 3(a); the coefficient of determination, $R^{2}$ is 0.9946$)$. In Eq. (5), the upper expression signifies that the mobility of the fully modified films is dominated by that of the surface species, which varies with $h_{0}$ according to Eq. (4). The lower expression is the same as Eq. (3), but with $h_{\mathrm{b}}$ set equal to zero and $h_{\mathrm{t}}$ replaced by $h_{\mathrm{UVO}}$. Thus, the UVO modified films with $h_{0}>h_{\mathrm{UVO}}$ can be regarded as 
bilayers having a surface layer of thickness $h_{\mathrm{UVO}}$ and mobility $M_{\mathrm{t}}$ on top and a bulk-like layer at the bottom, and the no-slip boundary condition applies. The fit values of $l_{\mathrm{t}}, \Delta l_{\mathrm{t}}, M_{\mathrm{t}}(\infty)$, and $h_{\mathrm{UvO}}$ are given in Table 1.

This fit result signifies that the rheological property of the top $14 \mathrm{~nm}$ of the films is modified by UVO, and its mobility is dominated by some surface species with total mobility $M_{\mathrm{t}}$.

Table 1. Summary of the model fit result of the UVO 1s PS451K films

\begin{tabular}{cccc}
\hline$l_{\mathrm{t}}(\mathrm{nm})$ & $\Delta l_{\mathrm{t}}(\mathrm{nm})$ & $M_{\mathrm{t}}(\infty)\left(\mathrm{nm}^{3} \cdot \mathrm{Pa}^{-1} \cdot \mathrm{s}^{-1}\right)$ & $h_{\mathrm{UVO}}(\mathrm{nm})$ \\
\hline $13.0 \pm 0.8$ & $0.94 \pm 0.20$ & $2.3 \times 10^{-4}$ & $14 \pm 2$ \\
\hline
\end{tabular}

Below this layer, the material is bulk-like. For the films with $h_{0}>h_{\mathrm{UvO}}$, the contribution of the bottom layer to $M_{\text {tot }}$ (which is given by $\left(h_{0}-h_{\mathrm{UvO}}\right)^{3} /\left(3 h_{\text {bulk }}\right)$ [13]) dominates except when $h_{0}-$ $h_{\mathrm{UVO}}<\sim 4 \mathrm{~nm}$, indicating that the UVO modified layer is more viscous than $\eta_{\text {bulk }}$ as inferred above. Equation (4) and the fit values of $l_{\mathrm{t}}$ and $\Delta l_{\mathrm{t}}$ reveal that $M_{\mathrm{t}}$ decreases with $h_{0}$ from $\sim 13 \mathrm{~nm}$ with a decay length of $\Delta l_{\mathrm{t}}=0.94 \mathrm{~nm}$. As discussed above, analogous $M_{\mathrm{t}}\left(h_{0}\right)$ dependences reported for PMMA [14] and PiBMA films supported by silica [19] might arise because of strong hydrogen bonds formed between the polymer's carboxylate groups and the substrate's surface silanol groups. The $M_{\mathrm{t}}\left(h_{0}\right)$ dependence of the UVO treated films thus corroborates with the previous finding [59] that UVO may cause formation of carboxylate groups in PS.

It should be mentioned that all of our $\eta_{\text {eff }}$ measurements were performed at temperatures well above the bulk $T_{\mathrm{g}}$, which may raise concerns about whether the oxygenated components 
caused by UVO might diffuse from the surface region to other regions of the films during our measurement. To address this concern, we observe that all the fit $\eta_{\text {eff }}$ values found of the films were constant with time beyond the rubbery elastic regime. Because the fit value of $\eta_{\text {eff }}$ is highly sensitive to how the mobile, bulk-like and slow components are distributed in the films, a time invariant $\eta_{\text {eff }}$ value suggests that diffusion of the oxygenated species did not take place in the terminal flow dynamic region. Such a finding is not inconsistent with the literature. It is reported that UVO treated PS (washed [52] or unwashed [60]) are stable for one month or longer. On the other hand, UVO treated poly(ethyleneterephthalate) (PET) - a more hydrophilic polymer demonstrated significantly inferior stability. Teare et al. [52] thus inferred that the stability found of UVO PS is caused by the hydrophobic nature of pristine PS, which deters the hydrophilic oxygenated surface species from diffusing into the hydrophobic bulk portion of the film. Klein et al. [51] studied the stability of 10 and $30 \mathrm{~s}$ UVO exposed PS and found that the surface oxygenated species diffused by no more than $1-2 \mathrm{~nm}$ from the surface upon annealing at $125^{\circ} \mathrm{C}$ (i.e., $\sim 25{ }^{\circ} \mathrm{C}$ above $T_{\mathrm{g}, \mathrm{bulk}}$ ) for $30 \mathrm{~min}$. These authors proposed crosslinking of the polymer by UVO besides hydrophobicity of the pristine polymer to explain the limited diffusion.

To support the idea that UVO treatment causes both chain scissioning and cross-linking of our polymer, the MW distributions of PS451K with and without UVO treatments were investigated by GPC. From the data presented in Fig. 6, the GPC spectrum broadens noticeably 


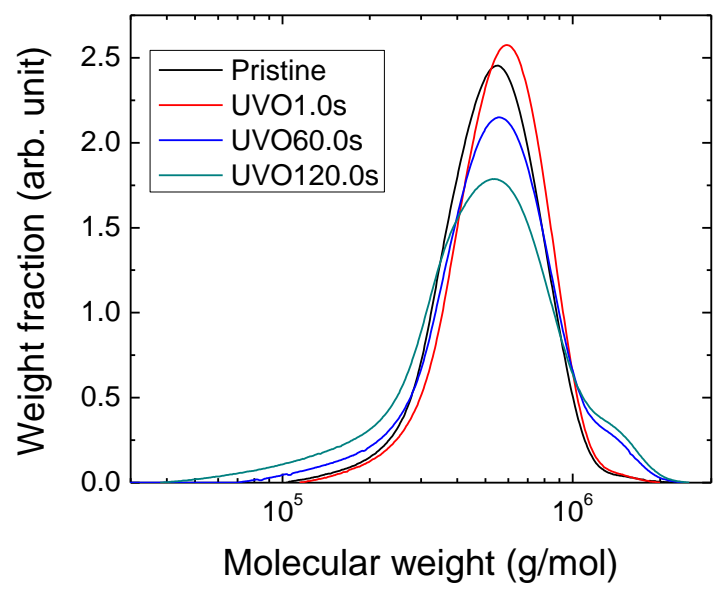

Fig. 6. Molecular weight distributions of pristine PS451K and PS451K after 1.0, 60.0 and $120.0 \mathrm{~s}$ UVO exposures.

as the UVO exposure time increases to $60.0 \mathrm{~s}$ or above. The observation of broadening on both sides of the spectrum is consistent with occurrence of both chain scissioning and cross-linking. In comparison, the $M_{\mathrm{w}}$ is much less affected. From the data, the PDI of the pristine polymer and the polymer after 1.0, 60.0 and $120.0 \mathrm{~s}$ UVO exposures are 1.16, 1.15, 1.27, and 1.43, respectively. Since the PDI values of the pristine and $1.0 \mathrm{~s}$ UVO-exposed samples are similar, the spatial extent of the UVO modifications relative to the sample thickness $(=1.6 \pm 0.6 \mu \mathrm{m})$ is minimal. The increase of PDI with UVO exposure time suggests that the spatial extent of UVO modification increases with the UVO exposure time. Because the penetration of UV into PS and other polymers is on the order of tens of nm or longer [61,62], our result suggests that the spatial extent of UVO modification may be limited by how far oxygen diffuses into the sample during the UVO exposure - a topic beyond the scope of this work, but one we are interested in.

Unlike the pristine films, the UVO-treated films with thickness below the $R_{\mathrm{g}} \approx 18 \mathrm{~nm}$ [50]) show no sign of interfacial slippage. The reason can be two-fold. Firstly, the PS polymer 
becomes less mobile upon UVO modification (as inferred from the higher viscosity of the UV modified layer), which deters the ability of the surface mobile component to induce plug flow in the film. Secondly, the depression demonstrated by $M_{\mathrm{t}}$ with decreasing $h_{0}$ (Fig. 3(a)) and the fact that $M_{\mathrm{tot}} \approx M_{\mathrm{t}}$ for $h_{0}<h_{\mathrm{UvO}}$ (Eq. (5)) suggest that the dynamics of the whole film becomes dominated by the substrate surface as carboxylate moieties and other oxygenated species are generated in the polymer, in keeping with the conclusions made before about the dynamics of the PMMA [14] and PiBMA films [19], where carboxylate groups are also present.

The finding that the $h_{\mathrm{UvO}}$ value $(=14 \pm 2 \mathrm{~nm})$ is comparable to the $R_{\mathrm{g}}$ of the polymer suggests that the former could originate from the latter. Specifically, with short UVO exposure times, the influence of the UVO modified species, even if the species are present within short distances of the surface, could pertain over longer distances up to $\sim R_{\mathrm{g}}$ by chain connectivity. To explore such an origin of the length scale of $h_{\mathrm{UvO}}$ and also that of $l_{\mathrm{t}}$, experiments are on-going to investigate the $\eta_{\mathrm{eff}}$ of UVO modified PS films with different $M_{\mathrm{w}}$ 's.

\section{Conclusion}

We report the influence of low-dose UVO treatment on the $\eta_{\text {eff }}$ of highly entangled PS $\left(M_{\mathrm{w}}=451 \mathrm{~kg} / \mathrm{mol}\right)$ films coated on silica. Before treatment, the $\eta_{\text {eff }}$ reduces as $h_{0}$ is decreased below $\sim 60 \mathrm{~nm}$. After a mere $1.0 \mathrm{~s}$ UVO exposure of the films under typical treatment conditions, the $\eta_{\text {eff }}$ increases rapidly as $h_{0}$ is decreased below $\sim 14 \mathrm{~nm}$. The data are described by a layer model assuming the UVO modified region to lie within $\sim 14 \mathrm{~nm}$ of the free surface and more viscous than the bulk polymer. Moreover, the mobility of this surface region is dominated by the mobility of a surface species that decreased with decreasing $h_{0}$, similar to the variations in 
surface mobility found with PMMA and PiBMA films on silica. For the UVO treated films with $h_{0}>\sim 14 \mathrm{~nm}$, the remaining portion of the films possesses a viscosity similar to that of the bulk polymer. Pristine PS nanometer films supported by silica are known to be unstable, and rupture spontaneously on heating even to temperatures below $T_{\mathrm{g}, \text { bulk }}$ [13]. Additionally, these films often exhibit dewetting rates much faster than predicted assuming the bulk viscosity [13, 15]. Our results show that the $\eta_{\text {eff }}$ of the most unstable film studied (where $h_{0} \approx 4 \mathrm{~nm}$ ) was increased by a factor of $\sim 10^{5}$ upon an UVO exposure of only $1.0 \mathrm{~s}$. In fact, in all the films we studied, no sign of dewetting was detectable even by AFM (n.b. The $3.5 \mathrm{~s} \mathrm{UVO} \mathrm{film,} \mathrm{with} h_{0}=10 \mathrm{~nm}$, was annealed at $172{ }^{\circ} \mathrm{C}$ for up to $4 \mathrm{~d}$.), corresponding to a significant improvement of the film's stability. In comparison, in the films stabilized by addition of nanofillers $[63,64]$ nucleated holes [64] or mesa-like structures [63] were visible already by optical microscopy upon annealing. The differences suggest different stabilization mechanisms. In our films, stabilization was achieved by suppression of roughening of the film surface, probably by virtue of the increases in $\eta_{\text {eff }}$ of the films with $h_{0}<\sim 14 \mathrm{~nm}$ (Fig. 3(b)). In the films stabilized by nanofiller addictives, on the other hand, stabilization was achieved by formation of a diffuse layer of the nanofillers at the substrate surface, which pins any three-phase contact line that may nucleate on the substrate surface [63, 64]. Importantly, this work shows that UVO treatment modifies the flow property of polymer films as well as improves their thermal stability. Given the low-cost and simplicity of the method, further explorations on the general applicability of UVO treatment to modulate the properties of polymer films are warranted.

\section{Acknowledgments}


We are indebted to Prof. Catherine Klapperich for letting us use the vacuum press in her lab. Support of the National Science Foundation through the grant award DMR-1310536 is gratefully acknowledged.

\section{References}

[1] J. A. Forrest, K. Dalnoki-Veress. The Glass Transition in Thin Polymer Films. Adv. Colloid Interface Sci. 94 (2001) 167-196.

[2] M. Alcoutlabi, G. B. McKenna. Effects of Confinement on Material Behavior at Nanometre Size Scale. J. Phys. Condens. Matter. 17 (2005) R461-R524.

[3] J. L. Keddie, R. A. L. Jones, R. A. Cory. Size-Dependent Depression of the Glass Transition Temperature in Polymer Films. Europhys. Lett. 27 (1994) 59-64.

[4] O. K. C. Tsui, T. P. Russell, C. J. Hawker. Effect of Interfacial Interactions on the Glass Transition of Polymer Thin Films. Macromolecules. 34 (2001) 5535-5539.

[5] O. K. C. Tsui, H. F. Zhang. Effect of Chain Ends and Chain Entanglement on Glass Transition Temperature of Polymer Thin Films. Macromolecules. 34 (2001) 9139-9142.

[6] D. S. Fryer, R. D. Peter, E. J. Kim, J. E. Tomaszewski, J. J. de Pablo, P. F. Nealey. Dependence of the Glass Transition Temperature of Polymer Films on Interfacial Energy and Thickness. Macromolecules. 34 (2001) 5627-5634. 
[7] P. Bernazzani, S. L. Simon, D. J. Plazek, K. L. Ngai. Effects of Entanglement Concentration on $\mathrm{T}_{\mathrm{g}}$ and Local Segmental Motions. Eur. Phys. J. E. 8 (2002) 201-207.

[8] R. D. Priestley, M. K. Mundra, N. J. Barnett, L. J. Broadbelt, J. M. Torkelson. Effects of Nanoscale Confinement and Interfaces on the Glass Transition Temperatures of a Series of Poly(N-Methacrylate) Films. Aust. J. Chem. 60 (2007) 765-771.

[9] J. E. Pye, C. B. Roth. Two Simultaneous Mechanisms Causing Glass Transition Temperature Reductions in High Molecularweight Freestanding Polymer Films as Measured by Transmission Ellipsometry. Phys. Rev. Lett. 107 (2011) 235701.

[10] B. Frank, A. P. Gast, T. P. Russell, H. Brown, C. Hawker. Polymer Mobility in Thin Films. Macromolecules. 29 (1996) 6531-6534

[11] J. M. Katzenstein, D. W. Janes, H. E. Hocker, J. K. Chandler, C. J. Ellison. Nanoconfined Self-Diffusion of Poly(Isobutyl Methacrylate) in Films with a Thickness-Independent Glass Transition. Macromolecules. 45 (2012) 1544-1552.

[12] X. Zheng, M. Rafailovich, J. Sokolov, Y. Strzhemechy, S. A. Schwarz. Long-Range Effects on Polymer Diffusion Induced by a Bounding Interface. Phys. Rev. Lett. 79 (1997) 241244.

[13] Z. Yang, Y. Fujii, F. K. Lee, C.-H. Lam, O. K. C. Tsui. Glass Transition Dynamics and Surface Layer Mobility in Unentangled Polystyrene Films. Science. 328 (2010) 1676-1679.

[14] R. N. Li, F. Chen, C.-H. Lam, O. K. C. Tsui. Viscosity of Polymethylmethacrylate on Silica: Epitome of Systems with Strong Polymer-Substrate Interactions. Macromolecules. 46 (2013) 7889-7893.

[15] F. Chen, D. Peng, C.-H. Lam, O. K. C. Tsui. Viscosity and Surface-Promoted Slippage of Thin Polymer Films Supported by a Solid Substrate. Macromolecules. 48 (2015) 5034-5039. 
[16] Y. Chai, T. Salez, J. D. McGraw, M. Benzaquen, K. Dalnoki-Veress, E. Raphael, J. A. Forrest. A Direct Quantitative Measure of Surface Mobility in a Glassy Polymer. Science. 343 (2013) 994.

[17] Z. Yang, A. Clough, C.-H. Lam, O. K. C. Tsui. Glass Transition Dynamics and Surface Mobility of Entangled Polystyrene Films at Equilibrium. Macromolecules. 44 (2011) 8294-8300.

[18] F. Chen, D. Peng, Y. Ogata, K. Tanaka, Z. Yang, Y. Fujii, N. L. Yamada, C.-H. Lam, O. K. C. Tsui. Effect of Confinement on the Effective Viscosity of Plasticized Polymer Films. Macromolecules. 48 (2015) 7719-7726.

[19] K. Geng, R. Katsumata, X. Yu, H. Ha, A. R. Dulaney, C. J. Ellison, O. K. C. Tsui. Conflicting Confinement Effects on the Tg, Diffusivity and Effective Viscosity of Polymer Films: A Case Study with Poly(Isobutyl Methacrylate) on Silica and Possible Resolution. (Submitted).

[20] M. P. Stoykovich, K. Yoshimoto, P. F. Nealey. Mechanical Properties of Polymer Nanostructures: Measurements Based on Deformation in Response to Capillary Forces. Appl. Phys. A. 90 (2008) 277-283.

[21] A. C. Mayer, S. R. Scully, B. E. Hardin, M. W. Rowell, M. D. McGehee. Polymer-Based Solar Cells. Materials Today. 10 (2007) 28-33.

[22] W. Yave, A. Car, J. Wind, K. V. Peinemann. Nanometric Thin Film Membranes Manufactured on Square Meter Scale: Ultra-Thin Films for Co2 Capture. Nanotechnology. 21 (2010) 395301.

[23] L. F. Villalobos, M. Karunakaran, K.-V. Peinemann. Complexation-Induced Phase Separation: Preparation of Composite Membranes with a Nanometer-Thin Dense Skin Loaded with Metal Ions. Nano Lett. 15 (2015) 3166-3171. 
[24] S. Napolitano. Staying Conductive in the Stretch. Science. 355 (2017) 24-25.

[25] M. D. Ediger, J. A. Forrest. Dynamics near Free Surfaces and the Glass Transition in Thin Polymer Films: A View to the Future. Macromolecules. 47 (2014) 471-478.

[26] P.-G. de Gennes. Glass Transitions in Thin Polymer Films. Eur. Phys. J. E. 2 (2000) 201205.

[27] T. Kajiyama, K. Tanaka, A. Takahara. Depth Dependence of the Surface Glass Transition Temperature of a Poly(Styrene-Block-Methyl Methacrylate) Diblock Copolymer Film on the Basis of Temperature-Dependent X-Ray Photoelectron Spectroscopy. Macromolecules. 28 (1995) 3482-3484.

[28] K. Tanaka, A. Taura, S.-R. Ge, A. Takahara, T. Kajiyama. Molecular Weight

Dependence of Surface Dynamic Viscoelastic Properties for the Monodisperse Polystyrene Film. Macromolecules. 29 (1996) 3040-3042.

[29] T. Kajiyama, K. Tanaka, A. Takahara. Surface Molecular Motion of the Monodisperse Polystyrene Films. Macromolecules. 30 (1997) 280-285.

[30] K. Tanaka, A. Takahara, T. Kajiyama. Effect of Polydispersity on Surface Molecular Motion of Polystyrene Films. Macromolecules. 30 (1997) 6626-6632.

[31] K. Tanaka, X. Jiang, K. Nakamura, A. Takahara, T. Kajiyama. Effect of Chain End Chemistry on Surface Molecular Motion of Polystyrene Films. Macromolecules. 31 (1998) 5148-5149.

[32] K. Tanaka, A. Takahara, T. Kajiyama. Rheological Analysis of Surface Relaxation Process of Monodisperse Polystyrene Films. Macromolecules. 33 (2000) 7588-7593. 
[33] F. Xie, H. F. Zhang, F. K. Lee, B. Du, O. K. C. Tsui, Y. Yokoe, K. Tanaka, A. Takahara, T. Kajiyama. Effect of Low Surface Energy Chain Ends on the Glass Transition Temperature of Polymer Thin Films. Macromolecules. 35 (2002) 1491-1492.

[34] Z. Fakhraai, J. A. Forrest. Measuring the Surface Dynamics of Glassy Polymers. Science. 319 (2008) 600-604.

[35] K. Paeng, S. F. Swallen, M. D. Ediger. Direct Measurement of Molecular Motion in Freestanding Polystyrene Thin Films. J. Am. Chem. Soc. 133 (2011) 8444-8447.

[36] C. J. Ellison, J. M. Torkelson. The Distribution of Glass-Transition Temperatures in Nanoscopically Confined Glass Formers. Nature Mater. 2 (2003) 695-670.

[37] W. Zhang, L. Yu. Surface Diffusion of Polymer Glasses. Macromolecules. 49 (2016) 731-735.

[38] L. Xie, G. B. DeMaggio, W. E. Frieze, J. DeVries, D. W. Gidley, H. A. Hristov, A. F. Yee. Positronium Formation as a Probe of Polymer Surfaces and Thin Films. Phys. Rev. Lett. 74 (1995) 4947-4950.

[39] S. Bekele, M. Tsige. Effect of Polymer/Solid and Polymer/Vapor Instantaneous Interfaces on the Interfacial Structure and Dynamics of Polymer Melt Systems. Langmuir. 32 (2016) 7151-7158.

[40] R. P. White, C. C. Price, J. E. G. Lipson. Effect of Interfaces on the Glass Transition of Supported and Freestanding Polymer Thin Films. Macromolecules. 48 (2015) 4132-4141.

[41] J. R. Vig. Uv /Ozone Cleaning of Surfaces. Journal of Vacuum Science and Technology A. 3 (1985) 1027-1034. 
[42] K. Efimenko, W. E. Wallace, J. Genzer. Surface Modification of Sylgard-184

Poly(Dimethyl Siloxane) Networks by Ultraviolet and Ultraviolet/Ozone Treatment. J. Colloid Interface Sci. 254 (2002) 306-315.

[43] Y. Berdichevsky, J. Khandurina, A. Guttman, Y. H. Lo. Uv/Ozone Modification of Poly(Dimethylsiloxane) Microfluidic Channels. Sensor Actuat B-Chem. 97 (2004) 402-408.

[44] I. Mathieson, R. H. Bradley. Improved Adhesion to Polymers by Uv/Ozone Surface Oxidation. International Journal of Adhesion and Adhesives. 16 (1996) 29-31.

[45] L. F. Macmanus, M. J. Walzak, N. S. McIntyre. Study of Ultraviolet Light and Ozone Surface Modificationof Polypropylene. J. Polym. Sci. B: Polym. Chem. 37 (1999) 2489-2501.

[46] D. O. H. Teare, N. Emmison, C. Ton-That, R. H. Bradley. Cellular Attachment to Ultraviolet Ozone Modified Polystyrene Surfaces. Langmuir. 16 (2000) 2818-2824.

[47] A. Bhattacharya, C. M. Klapperich. Mechanical and Chemical Analysis of Plasma and Ultraviolet-Ozone Surface Treatments for Thermal Bonding of Polymeric Microfluidic Devices. Lab on a Chip. 7 (2007) 876-882.

[48] G. V. Lubarsky, M. R. Davidson, R. H. Bradley. Elastic Modulus, Oxidation Depth and Adhesion Force of Surface Modified Polystyrene Studied by Afm and Xps. Surf. Sci. 558 (2004) $135-144$.

[49] J. M. Torres, C. M. Stafford, B. D. Vogt. Manipulation of the Elastic Modulus of Polymers at the Nanoscale: Influence of U V-Ozone Cross-Linking and Plasticizer. ACS Nano. 4 (2010) 5357-5365.

[50] L. J. Fetters, D. J. Lohse, S. T. Milner. Packing Length Influence in Linear Polymer Melts on the Entanglement, Critical, and Reptation Molecular Weights. Macromolecules. 32 (1999) 6847-6851. 
[51] R. J. Klein, D. A. Fischer, J. L. Lenhart. Systematic Oxidation of Polystyrene by

Ultraviolet-Ozone, Characterized by near-Edge X-Ray Absorption Fine Structure and Contact Angle. Langmuir. 24 (2008) 8187-8197.

[52] D. O. H. Teare, C. Ton-That, R. H. Bradley. Surface Characterization and Ageing of Ultraviolet-Ozone-Treated Polymers Using Atomic Force Microscopy and X-Ray Photoelectron Spectroscopy. Surface and Interface Analysis. 29 (2000) 276-283.

[53] D. Peng, Z. Yang, O. K. C. Tsui. Method to Measure the Viscoelastic Properties of Nanometer Entangled Polymer Films. Macromolecules. 44 (2011) 7460-7464.

[54] Z. Yang, C.-H. Lam, E. DiMasi, N. Bouet, J. Jordan-Sweet, O. K. C. Tsui. Method to Measure the Viscosity of Nanometer Liquid Films from the Surface Fluctuations. Appl. Phys. Lett. 94 (2009) 251906.

[55] C.-H. Lam, O. K. C. Tsui, D. Peng. Surface Dynamics of Noisy Viscoelastic Films by Adiabatic Approximation. Langmuir. 28 (2012) 10217-10222.

[56] H. Zhao, Y. J. Wang, O. K. C. Tsui. Dewetting Induced by Complete Versus Nonretarded Van Der Waals Forces. Langmuir. 21 (2005) 5817-5824.

[57] O. K. C. Tsui, Y. J. Wang, F. K. Lee, C.-H. Lam, Z. Yang. Equilibrium Pathway of SpinCoated Polymer Films. Macromolecules. 41 (2008) 1465-1468.

[58] L. J. Fetters, D. J. Lohse, T. A. Witten, A. Zirkel. Connection between Polymer Molecular Weight, Density, Chain Dimensions, and Melt Viscoelastic Properties. Macromolecules. 27 (1994) 4639-4647.

[59] S. A. Mitchell, A. H. C. Poulsson, M. R. Davidson, N. Emmison, A. G. Shard, R. H. Bradley. Cellular Attachment and Spatial Control of Cells Using Micro-Patterned UltraViolet/Ozone Treatment in Serum Enriched Media. Biomaterials. 25 (2004) 4079-4086. 
[60] B. W. Callen, M. L. Ridge, S. Lahooti, Neumann, R. N. S. Sodhi. Remote Plasma and Ultraviolet-Ozone Modification of Polystyrene. Journal of Vacuum Science and Technology A. 13 (1995) 2023-2029.

[61] R. Wilken, A. Hollander, J. Behnisch. Vacuum Ultraviolet Photolysis of Polyethylene, Polypropylene, and Polystyrene. Plasmas and Polymers. 7 (2002) 19-39.

[62] M. Strobel, M. J. Walzak, J. M. Hill, A. Lin, E. Karbashewski, C. S. Lyons. A Comparison of Gas-Phase Methods of Modifying Polymer Surfaces. Journal of Adhesion Science and Technology. 9 (1995) 365-383.

[63] M. E. Mackay, Y. Hong, M. Jeong, S. Hong, T. P. Russell, C. J. Hawker, R. Vestberg, J. F. Douglas. Influence of Dendrimer Additives on the Dewetting of Thin Polystyrene Films. Langmuir. 18 (2002) 1877-1882.

[64] K. A. Barnes, A. Karim, J. F. Douglas, A. I. Nakatani, H. Gruell, E. J. Amis. Suppression of Dewetting in Nanoparticle-Filled Polymer Films. Macromolecules. 33 (2000) 4177-4185. 


\title{
Modulation of the Effective Viscosity of Polymer
}

\section{Films by Ultraviolet Ozone Treatment}

\author{
Xuanji Yu, ${ }^{1,2}$ Anjeza Beharaj, ${ }^{3}$ Mark W. Grinstaff, ${ }^{3}$ Ophelia K. C. Tsui ${ }^{1,2} *$
}

Xuanji Yu, ${ }^{1,2}$ Anjeza Beharaj, ${ }^{3}$ Mark W. Grinstaff, ${ }^{3}$ Ophelia K. C. Tsui ${ }^{1,2,4 *}$

${ }^{1}$ Department of Physics, Boston University, Boston, MA 02215.

${ }^{2}$ Division of Materials Science and Engineering, Boston University, Boston, MA 02215.

${ }^{3}$ Department of Chemistry, Boston University, Boston, MA 02215.

${ }^{4}$ Department of Physics, Hong Kong University of Science and Technology, Clear Water Bay, Kowloon, HONG KONG.

*Correspondence to: okctsui@bu.edu.

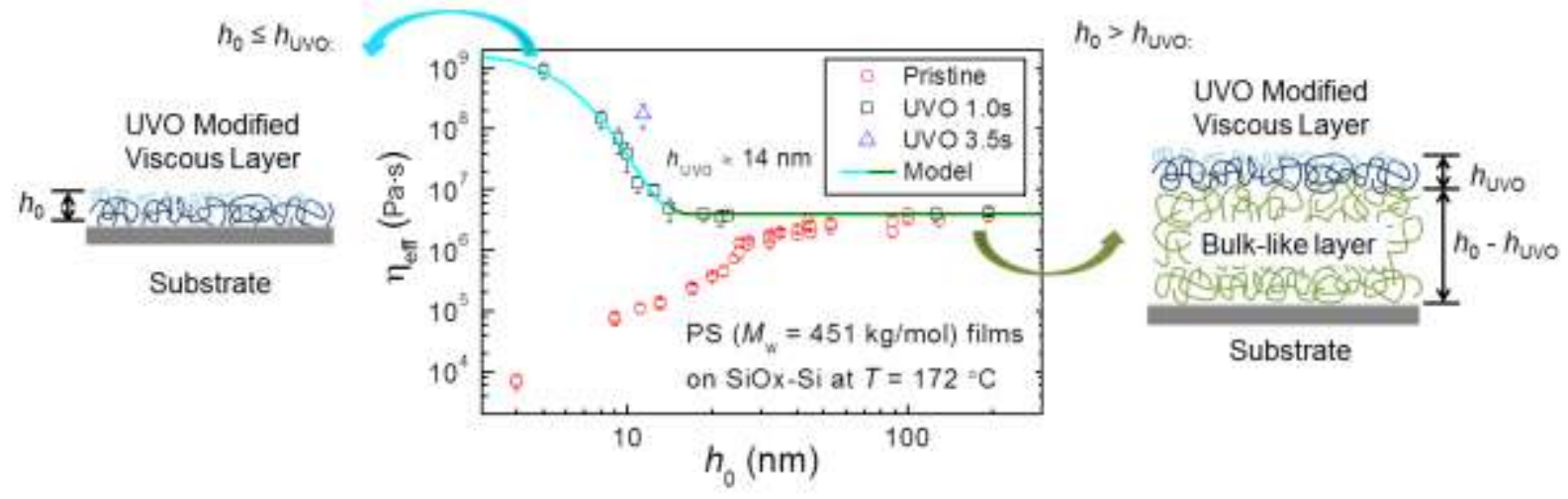

